\title{
ANÁLISE E DESENVOLVIMENTO DE UM RETIFICADOR HÍBRIDO MONOFÁSICO BIDIRECIONAL COM COMPENSAÇÃO SÉRIE NO BARRAMENTO CC PARA APLICAÇÕES EM MICRORREDES
}

\author{
Vítor F. Barbosa $^{1}$, Antônio O. C. Neto ${ }^{1}$, Gustavo B. Lima ${ }^{1}$, Danillo B. Rodrigues ${ }^{2}$ \\ ${ }^{1}$ Universidade Federal de Uberlândia-UFU, Núcleo de Pesquisa em Eletrônica de Potência-NUPEP, Uberlândia-MG, Brasil \\ ${ }^{2}$ Universidade Federal do Triângulo Mineiro-UFTM, Departamento de Engenharia Elétrica-DEE, Uberaba-MG, Brasil \\ e-mails: vitorfonsecabarbosa@gmail.com, gustavo.brito.28@gmail.com
}

\begin{abstract}
Resumo - O artigo proposto apresenta os trabalhos atrelados ao desenvolvimento de um Retificador Híbrido Monofásico Bidirecional composto por um retificador bidirecional (RNC/IPC) e um conversor SEPIC isolado. No lado de corrente alternada (CA) é realizada uma associação em paralelo a fim de assegurar a composição da corrente de entrada com reduzido conteúdo harmônico, tanto no modo de operação como retificador quanto como inversor. No lado de corrente contínua (CC) é realizada a associação série dos capacitores de filtro de saída a fim de assegurar a regulação de tensão no barramento $\mathrm{CC}$ e a divisão de contribuição de potência entre os conversores. $O$ desempenho do conversor proposto é avaliado no contexto de microrredes CC conectadas à rede $\mathrm{CA}$ de alimentação. A técnica de controle descentralizada desenvolvida promove a regulação do barramento $\mathrm{CC}$ da microrrede mesmo diante das intermitências inerentes às fontes de geração distribuída (GD), tais como a energia fotovoltaica. Os resultados teóricos e práticos apresentados neste trabalho evidenciam a eficácia da solução proposta.
\end{abstract}

Palavras-Chave - Compensação Série de Tensão, Correção Ativa do Fator de Potência, Microrredes, Retificador Híbrido, SEPIC.

\section{ANALISYS OF A SINGLE-PHASE HYBRID BIDIRECTIONAL RECTIFIER WITH A DC BUS SERIES VOLTAGE COMPENSATION FOR DC MICROGRIDS APPLICATION}

Abstract - The proposed article presents the works linked to the development of a Bidirectional Single-phase Hybrid Rectifier composed of a bidirectional rectifier (RNC/IPC) and an isolated SEPIC converter. On the alternating current $(A C)$ side, an association is made in parallel in order to ensure the composition of the input current with reduced harmonic content, both in the operation mode as rectifier and as inverter. On the direct current (DC) side, the serial association of the output filter capacitors is carried out in order to ensure the voltage regulation on the $d c$ bus and the division of power contribution between the converters. The performance of the proposed converter is evaluated in the context of dc

\footnotetext{
Manuscript received 05/04/2020; first revision 07/02/2020; accepted for publication 08/10/2020, by recommendation of Editor Demercil de Souza Oliveira Jr. http://dx.doi.org/10.18618/REP.2020.3.0027
}

microgrids connected to the ac supply network. The decentralized control technique developed promotes the regulation of the DC bus of the microgrid even in the face of the intermittencies inherent to distributed generation sources (GD), such as photovoltaic energy. The theoretical and practical results presented in this work show the effectiveness of the proposed solution.

Keywords - Active Power Factor Correction, Hybrid Rectifier, Microgrid, SEPIC, Series Voltage Compensation.

\section{NOMENCLATURA}

\begin{tabular}{|c|c|}
\hline Cl & Capacitor de saída do RNC/IPC. \\
\hline$C 2$ & Capacitor de saída do SEPIC. \\
\hline C3 & Capacitor série do SEPIC. \\
\hline$D_{\text {med }}$ & Razão cíclica média do SEPIC. \\
\hline$F_{\text {ond }}$ & $\begin{array}{l}\text { Frequência de ondulação de retificadores } \\
\text { em onda completa. }\end{array}$ \\
\hline Fs & $\begin{array}{l}\text { Frequência média de chaveamento para os } \\
\text { controladores de corrente por histerese. }\end{array}$ \\
\hline$\Delta I_{L 2}$ & Ondulação de corrente em L2. \\
\hline$\Delta I_{L m}$ & $\begin{array}{l}\text { Ondulação de corrente referida ao primário } \\
\text { em Lm. }\end{array}$ \\
\hline L1 & Indutor de entrada do RNC/IPC. \\
\hline$L 2$ & Indutor de entrada do SEPIC. \\
\hline$L m$ & $\begin{array}{l}\text { Indutância magnetizante do transformador } \\
\text { referida ao primário. }\end{array}$ \\
\hline$N p / N s$ & Relação de transformação. \\
\hline$P_{C A R G A}$ & Potência entregue a carga. \\
\hline$P_{R N C / I P C}$ & Potência processada pelo $R N C / I P C$. \\
\hline$P_{S E P I C}$ & Potência processada pelo SEPIC. \\
\hline$\triangle V_{R N C}$ & Ondulação de tensão na saída do RNC. \\
\hline$\triangle V_{\text {SEPIC }}$ & Ondulação de tensão na saída do SEPIC. \\
\hline$V_{C 1}, V_{R N C / I P C}$ & Tensão média de saída do RNC/IPC. \\
\hline$V_{C 2}, V_{S E P I C}$ & Tensão média de saída do SEPIC. \\
\hline$V_{I N \_P i c o}$ & Tensão de pico da entrada. \\
\hline$V_{I N \_R e t \_M e d}$ & Tensão retificada média de entrada. \\
\hline$V_{\text {OUT }}$ & Tensão média de saída do RHMB. \\
\hline$V_{Q 5}$ Pico & Tensão de pico no interruptor do SEPIC. \\
\hline$\omega$ & Frequência angular da rede. \\
\hline
\end{tabular}

\section{INTRODUÇÃO}

Com a crescente demanda por energia vinculada à preocupação em se utilizar modelos sustentáveis para o crescimento do setor energético, a distribuição de energia elétrica proveniente de sistemas de fontes de Geração Distribuída (GD) tem crescido significativamente nos 
últimos anos. São sistemas mais eficientes quando comparados aos sistemas centralizados, os quais apresentam elevadas perdas de transmissão, preocupações ambientais de implementação de grandes usinas, dentre outros. Neste contexto, a adição de fontes renováveis de energia próximas às unidades consumidoras, a integração de cargas a um barramento de alimentação comum aliados a controles robustos e estáveis estão relacionados ao conceito de Microrredes (MR) [1].

As MRs são capazes de unificar um sistema local em baixa e média tensão com diversas cargas, tendo como principal elemento as fontes de GD. Estas se integram a então existente rede elétrica como um único ponto visto pelo Sistema Elétrico de Potência, ou também podem operar de forma autônoma e desconectada à rede elétrica [2]. A Figura 1 ilustra um arranjo típico de uma MR CC.

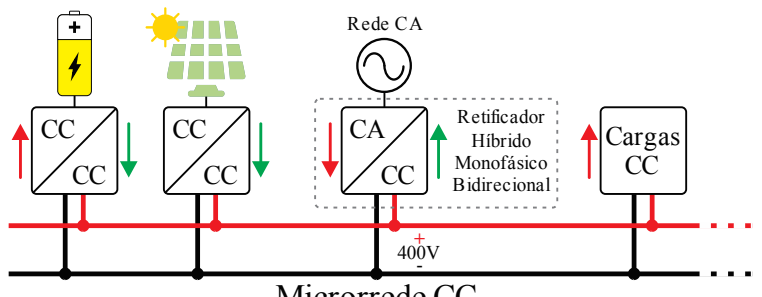

Microrrede CC

Fig. 1. Esquema típico de uma Microrrede $\mathrm{CC}$ conectada à rede.

A MR em CA possui algumas vantagens devido a todo aparato tecnológico de dispositivos de proteção, normas e especificações já estabelecidas em sistemas de distribuição de energia elétrica convencionais. Porém, há problemas de sincronização entre as fontes de microgeração e a rede elétrica, eventuais desequilíbrios de fases, complexidade no controle de potência reativa, além da adição de mais estágios de conversão, o que pode reduzir a eficiência do sistema.

Em contrapartida, as MRs CC possuem maior eficiência, confiabilidade e estabilidade, pois possibilitam uma conexão direta a vários equipamentos e cargas operando em $\mathrm{CC}$, como por exemplo, sistemas de acionamento de máquinas elétricas através de circuitos ASD (ajustable speed driver), sistemas de iluminação moderna por LED, recarga rápida de baterias de veículos elétricos, dentre outros. Desta forma, a redução dos custos na implementação de estruturas de sistemas de GD é alcançada e possibilita a inserção destes tipos de fontes [3]-[8], expandindo assim, de forma descentralizada a microgeração.

Além das MR CC unificarem diversos tipos de carga em um barramento $\mathrm{CC}$ comum, uma parte importante de sua composição é o conversor de interface com a rede elétrica em CA. Este conversor é responsável por garantir a regulação de tensão no barramento, assim como uma corrente em formato senoidal com baixa Distorção Harmônica Total de Corrente (DHTi), por isso, diversos estudos buscam uma topologia que apresente melhor rendimento/eficiência e confiabilidade. A partir da necessidade de estruturas capazes de processar uma grande quantidade de potência, a associação de conversores em paralelo se mostra como uma boa opção para aumentar a densidade de potência das MR [9].

Estudos recentes sobre topologias de retificadores híbridos têm demonstrado sua viabilidade para aplicações em MR $\mathrm{CC}$, uma vez que possuem alta densidade de potência e robustez inerente a própria topologia [10]-[17]. Esta família de retificadores híbridos contemplam os principais requisitos adotados para um conversor de interface com a rede, com isso, algumas configurações se destacam por serem potenciais alternativas nestas aplicações, visto que oferecem um alto Fator de Potência (FP) e reduzida DHTi, além de regular o barramento $\mathrm{CC}$ comum a um nível fixo préestabelecido.

Vale destacar que os retificadores que apresentam a característica de compensação série de tensão no barramento CC se destacam pela confiabilidade devido ao fato de serem imunes a afundamentos de tensão, assim como desequilíbrios de fase na rede $\mathrm{CA}$ e oscilações de tensão devido às intermitências de alguns dos sistemas de GD.

Dentre as diversas técnicas de controle associadas aos conversores de uma MR, destaca-se o controle descentralizado [18], uma vez que sua simplicidade, robustez e ausência de comunicação com outros conversores que compõem a MR é garantida. Desta forma, controladores como o Proporcional Integral (PI) já são empregados em estruturas híbridas e vão ao encontro das exigências impostas pelas MRs CC.

Neste contexto, com a premissa de unificar os benefícios de se empregar estruturas topológicas de elevado rendimento e confiabilidade com técnicas de controle robustas e descentralizadas, um método conhecido como DC Bus Signaling [19] busca informações de operação apenas no barramento CC da MR. Essa estratégia segue os mesmos princípios que nortearam o desenvolvimento da técnica apresentada preliminarmente pelos autores deste trabalho em [20]. Dando continuidade aos trabalhos, nesta oportunidade são apresentadas importantes contribuições no que tange ao projeto e dimensionamento dos conversores estáticos que compõem o grupo retificador; ao projeto e implementação da estratégia de controle descentralizado para regulação da tensão no barramento $\mathrm{CC}$ da $\mathrm{MR}$; e toda a etapa de implementação do protótipo e sua avaliação experimental em laboratório.

Conforme observado na Figura 2, o Retificador Híbrido Monofásico Bidirecional (RHMB) em pauta é composto por um retificador bidirecional (RNC/IPC) e um conversor SEPIC isolado. No lado CA é realizada uma associação em paralelo a fim de assegurar a composição da corrente de entrada com reduzido conteúdo harmônico -tanto no modo de operação como retificador, quanto como inversor- e reduzir os esforços de corrente nos elementos passivos e semicondutores. No lado CC é realizada a associação série dos capacitores de filtro de saída a fim de assegurar a regulação de tensão no barramento $\mathrm{CC}$ da $\mathrm{MR}$ e a divisão de contribuição de potência entre os conversores. Adicionalmente, os esforços de tensão nos elementos passivos e nos semicondutores são reduzidos. Destaca-se ainda que poucos sensores são utilizados ( 01 de corrente e 02 de tensão), reduzindo custos e aumentando a confiabilidade da estrutura. Para compor a MR CC, além das cargas em corrente contínua, foi introduzido um gerador fotovoltaico cuja interface com a MR é realizada através de um conversor Boost clássico operando com técnica de rastreamento do ponto de máxima potência, os quais não são o foco deste trabalho. 


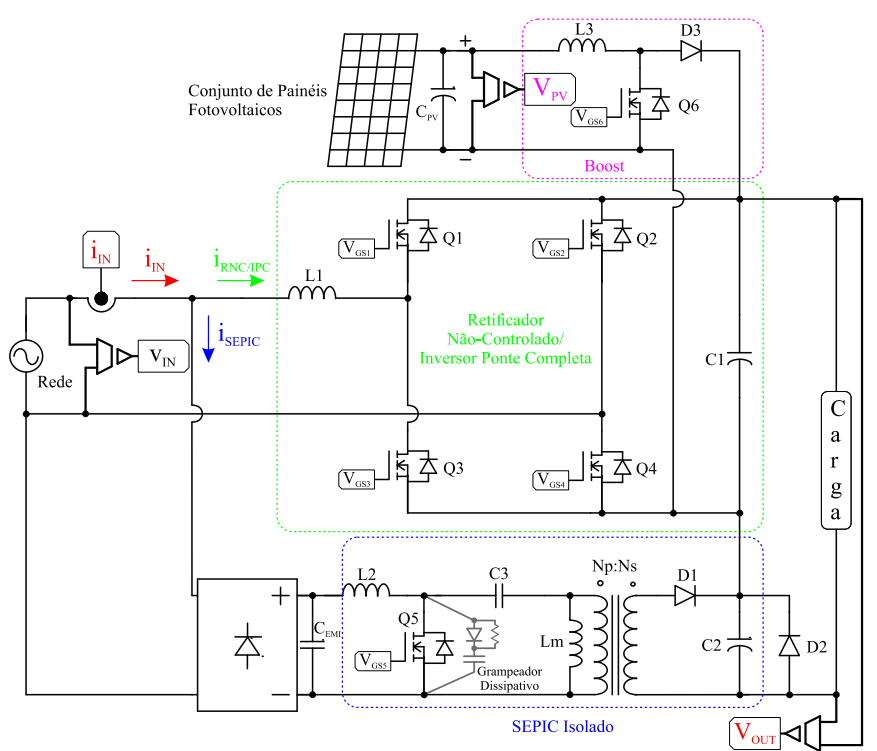

Fig. 2. Estrutura topológica proposta.

\section{ESTRUTURA PROPOSTA}

Para compreensão da solução proposta, os seguintes tópicos são abordados nesta seção: (A) Princípio de Funcionamento; (B) Dimensionamento da Estrutura RHMB; (C) Estratégia de Controle e Projeto dos Controladores.

\section{A. Principio de Funcionamento}

O RHMB opera em dois modos distintos (retificação e inversão). Na retificação, quando as fontes de GD (neste caso representada por conjunto de módulos fotovoltaicos (PV) e um conversor Boost) fornecem uma energia menor que a consumida pela carga, os pulsos de ativação dos interruptores do RNC/IPC são desativados para fazê-lo operar como um retificador não-controlado convencional. Os elementos L1 e C1 funcionam como um filtro em baixa frequência tornando a saída estabilizada em um nível de tensão praticamente fixo (ondulação desprezível).

A associação série de tensão na saída torna possível a operação do conversor SEPIC com esforços reduzidos nos semicondutores. Nesse sentido, para os níveis de tensão definidos, a tensão ideal de bloqueio do interruptor de potência Q5 do SEPIC leva em consideração a tensão máxima após a ponte retificadora e a tensão regulada na saída do conversor referida ao primário, a qual é expressa por (1) [21]. Todavia, a adição de circuitos grampeadores dissipativos se faz necessária para atenuar a tensão de pico inerente a essas estruturas com transformadores armazenadores de energia, como ocorre em conversores do tipo flyback.

$$
V_{Q 5_{-} \text {Pico }}=V_{I N_{-} \text {Pico }}+V_{\text {SEPIC }} \times \frac{N p}{N S} .
$$

Ainda na operação como retificador, o SEPIC é capaz de realizar a Correção do Fator de Potência (CFP), além de regular a tensão no elo $\mathrm{CC}$. Sua isolação é necessária para prevenir curto-circuito na compensação série de tensão no barramento. Todavia, cabe salientar que não há a isolação galvânica entre a MR CC e a rede CA.
Uma vez reconhecida a operação do RNC em baixa frequência e, as etapas de operação de um SEPIC isolado [21], as Figuras 3 e 4 têm como foco principal a compreensão do processo de composição de tensões e correntes na etapa de saída do RHMB. É possível perceber que a corrente $\mathrm{I}_{\mathrm{CARGA}}$ é comum às duas saídas dos conversores (RNC/IPC e SEPIC). Na condição de intermitência da GD, a relação do processamento de potência entre os conversores é definida pela proporção da tensão regulada no barramento CC [16]. Sendo assim, idealmente tem-se:

$$
\begin{gathered}
P_{R N C / I P C}=\frac{V_{R N C / I P C}}{V_{\text {OUT }}} \times P_{C A R G A}=\frac{V_{C 1}}{V_{\text {OUT }}} \times P_{C A R G A} \\
P_{S E P I C}=\frac{V_{S E P I C}}{V_{\text {OUT }}} \times P_{C A R G A}=\frac{V_{C 2}}{V_{\text {OUT }}} \times P_{C A R G A} .
\end{gathered}
$$

Portanto, observa-se que a parcela de potência a ser processada por cada conversor pode ser determinada de acordo com a tensão média nos capacitores $\mathrm{C} 1$ e C2. Uma vez que a tensão média no capacitor $\mathrm{C} 1$ tem relação direta com a tensão de pico da rede CA de alimentação, por consequência, para determinação do valor médio de tensão em C2 $\left(\mathrm{V}_{\text {SEPIC }}\right)$,

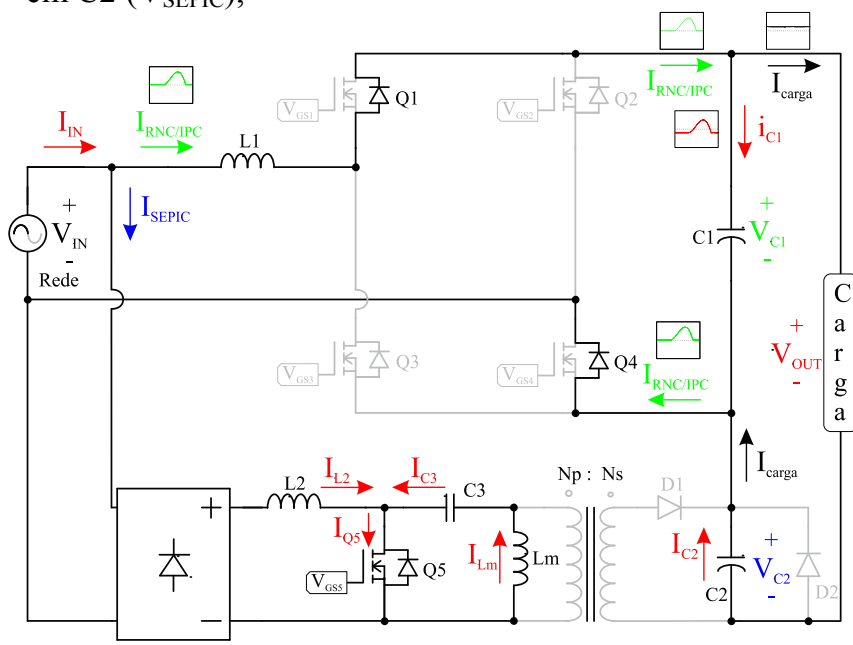

Fig. 3. Etapa de operação: Interruptor de potência Q5 ligado.

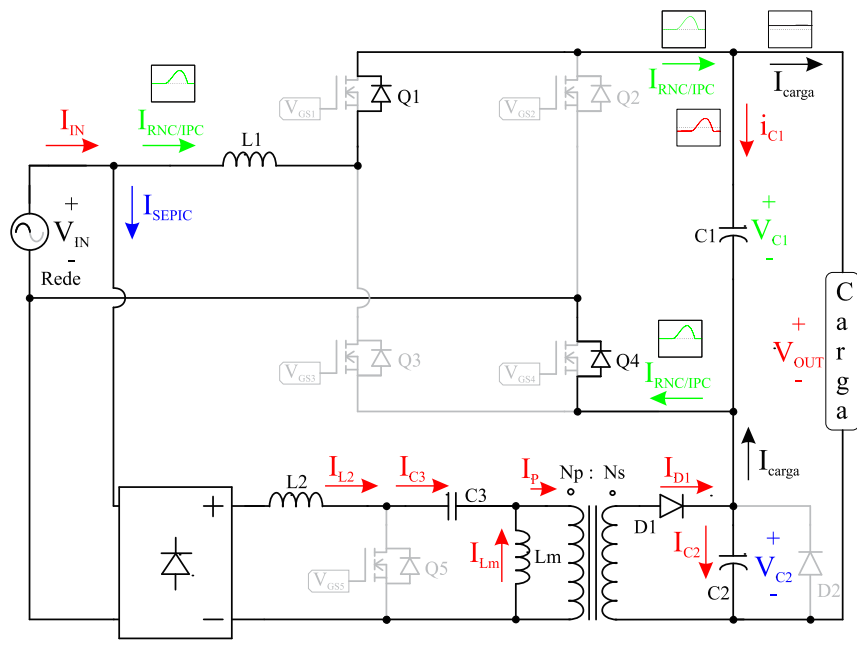

Fig. 4. Etapa de operação: Interruptor de potência Q5 desligado. 
considera-se a diferença entre a tensão média no barramento $\mathrm{CC}$ da MR ( $\left.\mathrm{V}_{\text {OUT }}\right)$ e a tensão média em C1 ( $\left.\mathrm{V}_{\mathrm{RNC} / \mathrm{IPC}}\right)$.

Para o modo de inversão, quando as fontes de GD fornecem uma quantidade de energia maior que a demandada pela carga, o conversor SEPIC -que faz a conversão unidirecional do fluxo de potência- é desativado, uma vez que o diodo D2 entra em condução. Dessa maneira, o RNC/IPC passa a operar como um inversor ponte completa de interface com a rede elétrica processando apenas a energia excedente proveniente da GD. A escolha do indutor de filtro L1 de primeira ordem confere a estrutura maior robustez, uma vez que capacitores apresentam maiores desgastes e taxas de falha. Por fim, um conversor do tipo Boost com o capacitor $\mathrm{C} 1$ compartilhado com o RNC/IPC é utilizado como interface de condicionamento da energia proveniente da GD. Um capacitor denominado $\mathrm{C}_{\mathrm{PV}}$ é utilizado na saída do gerador fotovoltaico para reduzir as oscilações em tensão e, por consequência, a ondulação de potência processada pelo Boost [22].

\section{B. Dimensionamento da Estrutura RHMB}

$\mathrm{O}$ dimensionamento dos elementos passivos do RHMB é realizado na condição de intermitência da GD (no modo retificador), visto que, a ondulação de tensão em $\mathrm{C} 1$ é maior por operar em baixa frequência e o SEPIC tem seu funcionamento apenas no modo de retificação.

$\mathrm{O}$ indutor L1 opera no regime de condução descontínuo, como indicado na Figura 3, seu dimensionamento baseia-se na solução numérica de equações para definir os instantes $t_{1} \mathrm{e}$ $\mathrm{t}_{4}$, aplicando-os em (4) [23].

$$
\begin{array}{r}
L 1=\frac{-V_{R N C / I P C \times F_{\text {ond }}}}{P_{R N C}}\left\{\frac{V_{I N_{-} P i c o}\left[\operatorname{sen}\left(\omega t_{4}\right)-\operatorname{sen}\left(\omega t_{1}\right)\right]}{\omega^{2}}+\right. \\
\left.\frac{V_{I N_{\text {P Pico }}}\left[\cos \left(\omega t_{1}\right) \times\left(t_{4}-t_{1}\right)\right]}{\omega}+\frac{V_{R N C / I P C}\left(t_{4}{ }^{2}+t_{1}{ }^{2}\right)}{2}-V_{R N C / I P C} t_{1} t_{4}\right\} .
\end{array}
$$

Já a determinação de $\mathrm{C} 1$ é realizada através de (5).

$$
C 1=\frac{P_{R N C / I P C}}{2 \times V_{R N C / I P C} \times \Delta V_{R N C / I P C^{\times}} \times F_{\text {ond }}} .
$$

O primeiro passo para determinação dos elementos passivos do SEPIC é a definição da razão cíclica média de operação, descrita por (6). A tensão média de entrada do SEPIC é a mesma observada em circuitos retificadores de onda completa com carga resistiva.

$$
\frac{V_{S E P I C}}{V_{I N_{-} R e t_{-} M e d}}=\frac{D_{\text {med }}}{1-D_{\text {med }}} \times \frac{N_{S}}{N_{P}} .
$$

Logo em seguida, o espectro de chaveamento do controlador de corrente por histerese é variado e delimitado pela metade da frequência de amostragem, ou seja, $40 \mathrm{kHz}$ [16]. Desta maneira, a escolha da frequência de chaveamento média $\left(\mathrm{F}_{\mathrm{S}}\right)$ é adotada como $25 \mathrm{kHz}$, um valor abaixo da máxima possível.

$\mathrm{O}$ indutor L2 e a indutância magnetizante do transformador referida ao primário $(\mathrm{Lm})$, são dimensionados por (7) e (8). É importante notar que a ondulação de corrente em L2 não pode ser elevada, uma vez que a DHTi depende desta característica.

$$
\begin{aligned}
& L 2=\frac{V_{I N_{-} R e t_{\text {Med }}} \times D_{\text {med }}}{\Delta I_{L 2} \times F s} \\
& L m=\frac{\left(V_{S E P I C} \times \frac{N p}{N s}\right)}{\Delta I_{L m} \times F s} \times\left(1-D_{\text {med }}\right) .
\end{aligned}
$$

Para a devida operação do SEPIC na CFP, o capacitor série C3 não é dimensionado de acordo com sua ondulação de tensão. Este capacitor precisa seguir o comportamento em baixa frequência $(120 \mathrm{~Hz})$ da tensão retificada, com isso, recomenda-se o ajuste por simulação computacional [24]. Valores usuais para esta aplicação estão na faixa dos 1 a $4,7 \mu \mathrm{F}$.

Por fim, de maneira similar ao cálculo de $\mathrm{C} 1$, o capacitor C2 de saída do SEPIC precisa filtrar a componente fundamental da tensão retificada da rede. $O$ cálculo é realizado através de (9).

$$
C 2=\frac{P_{S E P I C}}{\omega \times V_{S E P I C} \times \Delta V_{S E P I C}} .
$$

\section{Estratégia de Controle e Projeto dos Controladores}

A estratégia de controle foi implementada em linguagem C e embarcada no DSP da Texas Instruments ${ }^{\circledR}$ (TMS320F28379D), que apresenta como uma de suas grandes vantagens o $D A C$ embutido que permite análises em tempo real do controle interno discretizado. A taxa de amostragem do algoritmo é de $80 \mathrm{kHz}$. A Figura 5 ilustra o esquema de controle implementado.

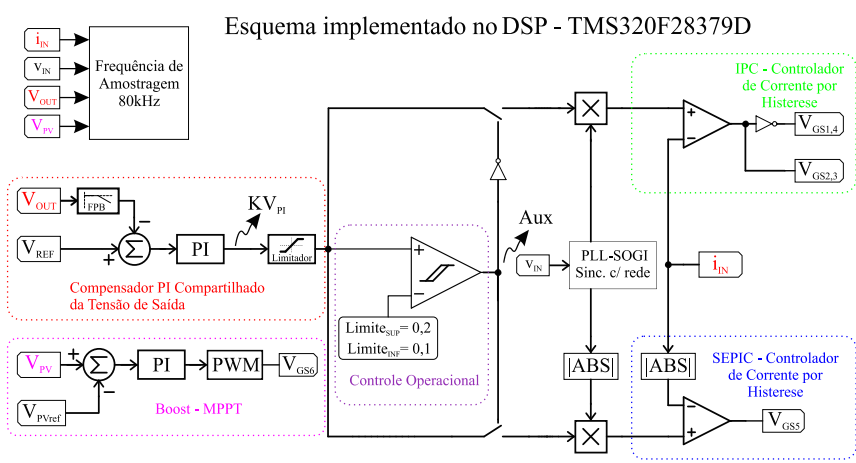

Fig. 5. Estratégia de controle proposta.

Para operação da estrutura com alto FP, é de grande importância a utilização de uma referência atracada em frequência e fase com o sinal da rede elétrica e que esta seja imune a ruídos. O algoritmo de Phase Locked Loop (PLL) do tipo $S O G I$ se mostra superior em relação aos demais quanto a transitórios no sistema, além de baixas oscilações na referência gerada [25].

O controle da GD é realizado por meio de um algoritmo de rastreamento do ponto de máxima potência em tensão constante devido a sua simplicidade e facilidade de implementação, uma vez que a eficiência nesse rastreamento do gerador fotovoltaico não é o foco deste trabalho.

Por fim, o comparador de histerese recebe um sinal proporcional $\left(\mathrm{KV}_{\mathrm{PI}}\right)$ proveniente do PI. Como este sinal é, de fato, a referência para os controladores de corrente, ele representa a corrente de pico em cada ciclo e também a potência CA vista pela entrada [26]. Desta forma, o 
comparador de histerese interpreta se a estrutura RHMB opera no modo de retificação quando $\mathrm{KV}_{\mathrm{PI}}$ é positivo, ou no modo de inversão, quando está em um nível abaixo de 0,1 . A fim de promover estabilidade na operação do sistema, foi definida uma zona morta na transição entre os modos de operação como retificação e inversão, onde $K V_{P I}$ está entre os limiares de 0,1 e 0,2. Assim a estrutura se torna imune às oscilações

naturais do PI mediante condições de baixa potência drenada da rede CA, ou seja, quando as fontes renováveis fornecem quase toda a energia demandada pela carga. A porta inversora localizada após o comparador confere o intertravamento entre os pulsos de gatilho dos interruptores dos conversores SEPIC e RNC/IPC.

Posto que a imposição de corrente de entrada se dá a partir da comparação direta entre a corrente de entrada e sua respectiva referência senoidal (Controlador Histerese), não é necessário o projeto de um controlador para a malha de corrente. Portanto, o objetivo principal da metodologia de projeto se volta para a especificação do controlador de tensão da MR. Assim, o diagrama de blocos do sistema equivalente do RHMB (SEPIC+RNC/IPC) pode ser representado conforme ilustrado na Figura 6, em que permite-se projetar o controlador de tensão com base na análise da função de transferência equivalente do sistema em malha aberta $\mathrm{G}_{v i \text { eq }}(\mathrm{s})$, a qual relaciona a tensão controlada no barramento $\mathrm{CC}\left(\mathrm{V}_{\text {OUT }}(\mathrm{s})\right.$ - variável controlada) com a manipulação da amplitude da corrente da rede CA de alimentação ( $\left.i_{\text {in }}\right)$ imposta a partir da variação da amplitude da referência senoidal de corrente $\left(\mathrm{I}_{\mathrm{REF}}(\mathrm{s})\right.$ - variável manipulada).

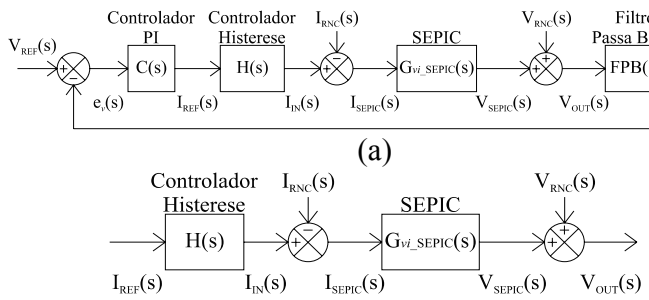

(b)

Fig. 6. Diagramas de blocos do sistema equivalente (a) do conversor RHMB em malha fechada e (b) do sistema equivalente em malha aberta, representado por $\mathrm{G}_{v i \text { eq }}(\mathrm{s})$.

Para levantamento da função de transferência do sistema no modo retificação, utilizou-se a plataforma PSIM $^{\circledR}$ para implementação do controlador de corrente utilizado no acionamento do interruptor do conversor SEPIC $\left(Q_{5}\right)$, conforme ilustrado na Figura 7. Um degrau na referência de corrente de amplitude $2 \mathrm{~A}\left(\mathrm{I}_{\mathrm{REF} \text { (inicial) }}\right)$ para $5 \mathrm{~A}\left(\mathrm{I}_{\mathrm{REF}(\text { final })}\right)$ no instante $0,5 \mathrm{~s}$ foi imposto a fim de se verificar o comportamento da resposta da tensão de saída $\left(\mathrm{V}_{\text {OUT }}\right)$ e da corrente de entrada $\left(\mathrm{i}_{\mathrm{IN}}\right)$. Os resultados obtidos são apresentados na Figura 8.

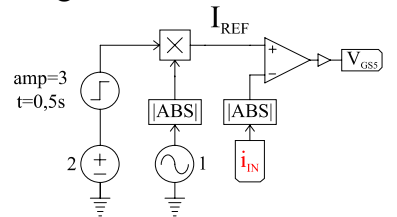

Fig. 7. Controlador de corrente utilizado no ensaio de resposta transitória do sistema.
De posse dos resultados apresentados na Figura 8, percebe-se que a resposta do sistema em malha aberta pode ser aproximada a uma resposta de sistemas de primeira ordem, podendo ser obtidas as informações necessárias para se estimar a função de transferência do Sistema Equivalente $\mathrm{G}_{v i \text { eq }}(\mathrm{s})$, a saber: os valores médios de $\mathrm{V}_{\text {OUT }}$ em regime permanente antes e após a perturbação ( $\mathrm{V}_{\text {OUT(inicial) }} \mathrm{e}$ $\mathrm{V}_{\text {OUT(final) }}$ ) e o tempo necessário para a resposta da tensão $\mathrm{V}_{\text {OUT }}$ atingir $63 \%$ do seu valor em regime permanente $\left(\mathrm{T}_{(63 \%)}\right)$. Dessa forma, tem-se que:

$$
G_{v i \_ \text {eq }}(s)=\frac{V_{\text {OUT }}(s)}{I_{R E F}(s)}=k \frac{a}{s+a},
$$

sendo:

$$
\begin{gathered}
k=\frac{V_{\text {OUT }(\text { final })}-V_{\text {OUT (inicial })}}{I_{R E F(\text { final })}-I_{R E F(\text { inicial })}} \\
a=\frac{1}{T_{(63 \%)}}
\end{gathered}
$$

onde:

a - frequência do polo do sistema de primeira ordem.

$k$ - ganho do sistema de primeira ordem.

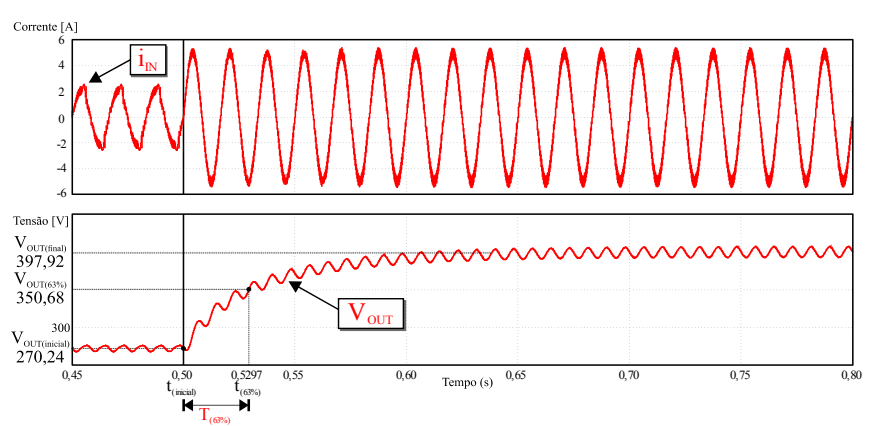

Fig. 8. Resposta transitória da corrente de entrada e tensão de saída do RHMB.

Dessa forma, o diagrama de controle em malha fechada do sistema pode ser representado conforme ilustrado na Figura 9. A função de transferência do Sistema Equivalente é dada por (13). Já o filtro passa baixa de ganho $K_{f}$ unitário com frequência angular de corte $\omega_{c}$ em 75,4 rad/s é representado por (14).

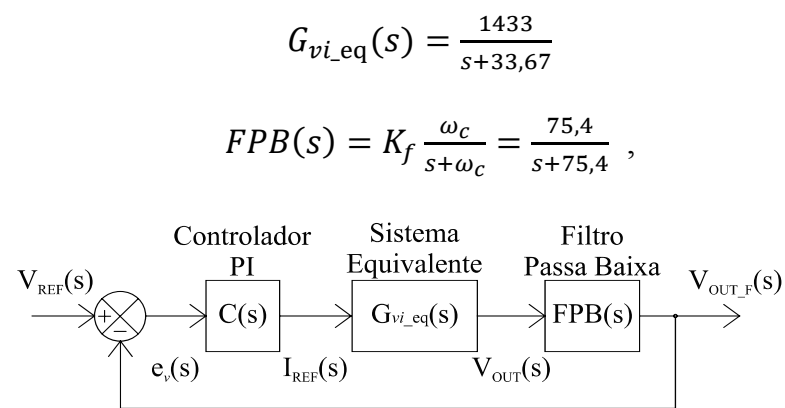

Fig. 9. Diagrama de blocos do sistema equivalente aproximado do conversor RHMB em malha fechada.

Utilizando o software Scilab®, simulou-se o sistema em malha fechada sem compensação $(\mathrm{C}(\mathrm{s})=1)$ para verificar a característica da resposta ao degrau e determinar as 
melhorias necessárias nas respostas transitória e em regime permanente. A Figura 10 ilustra a resposta ao degrau unitário do sistema em malha fechada sem compensação, bem como os diagramas de Bode em malha aberta. Verifica-se que a resposta do sistema é rápida, apresentando um reduzido tempo de acomodação (69,2ms). Entretanto, a ultrapassagem percentual (UP) da resposta do sistema é muito alta (59\%), em decorrência da baixa margem de fase do sistema $\left(19,1^{\circ}\right.$ na frequência de $51,5 \mathrm{~Hz}$ ).

Deste modo projetou-se um controlador PI capaz de reduzir a UP da resposta do sistema para um valor abaixo de $10 \%$, garantindo uma margem de fase maior que $45^{\circ}$, bem como um tempo de acomodação na ordem de $100 \mathrm{~ms}$. A função de transferência do controlador PI é representada por (15) e a Figura 11 ilustra a resposta ao degrau unitário do sistema em malha fechada compensado, bem como os diagramas de Bode em malha aberta. Verifica-se que a resposta do sistema se manteve rápida, apresentando um tempo de acomodação de $105 \mathrm{~ms}$, com uma UP de $8,33 \%$, garantida pela margem de fase de $57,3^{\circ}$.

$$
C(s)=0,07 \frac{(0,05 s+1)}{0,05 s}=0,07 \frac{(s+20)}{s} .
$$

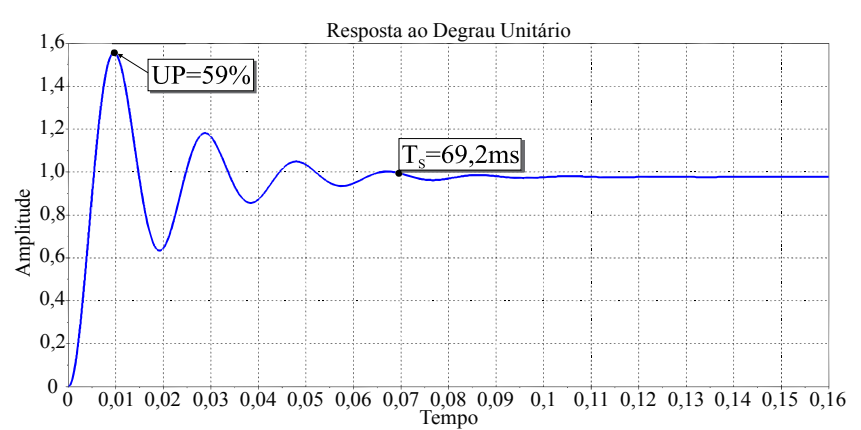

(a)
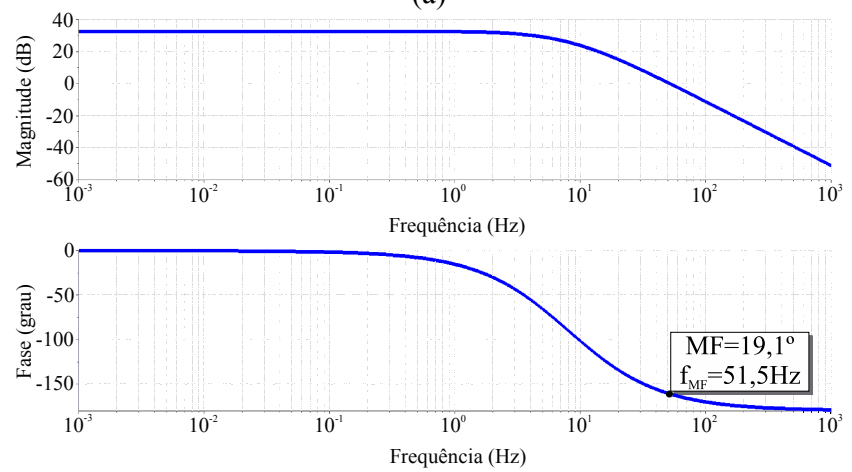

(b)

Fig. 10. (a) Resposta ao degrau unitário do sistema em malha fechada sem compensação (b) diagramas de Bode em malha aberta.

Por fim, foram realizadas simulações no software PSIM ${ }^{\circledR}$, variando-se os parâmetros (ganho $\mathrm{K}_{\mathrm{PI}}$ e constante de tempo $\mathrm{T}_{\mathrm{PI}}$ ) do compensador tornando possível a obtenção de um ábaco -apresentado na Figura 12- que correlaciona os valores de ganho K do compensador com os valores de DHTi e UP. Conclui-se que os valores de ganho têm forte influência sobre a DHTi em regime permanente e isso ocorre pois o ganho do PI é responsável por atenuar a ondulação em $120 \mathrm{~Hz}$, a qual é multiplicada pela referência de corrente retificada. Por outro lado, a constante de tempo está atrelada a UP após a perturbação. A norma IEC 61000-3-2 não delimita níveis de DHTi (apenas níveis individuais de componentes harmônicos), todavia, uma operação abaixo dos $5 \%$ garante a adequação à norma. Desta maneira, a curva em azul foi a escolhida por apresentar uma UP moderada em torno de $10 \%$ e uma DHTi de 5,0\%. Após a realização de testes práticos, realizou-se uma sintonia fina no controlador PI projetado, sendo implementado na prática com a função de transferência representada por (16).

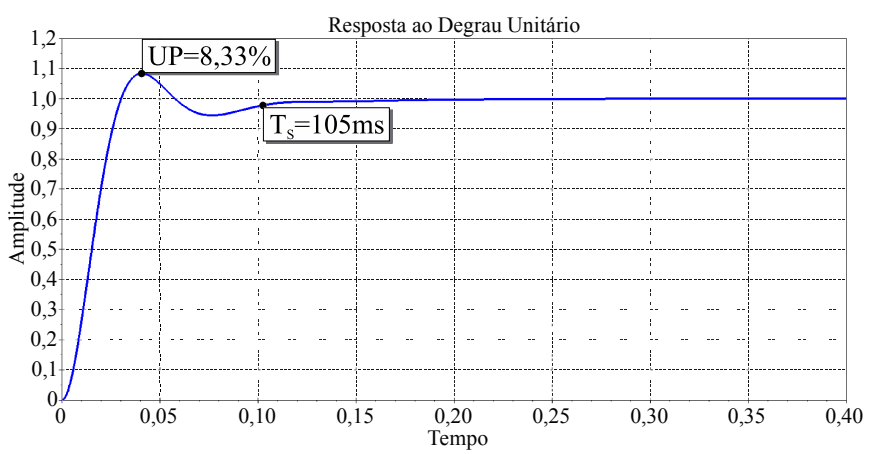

(a)
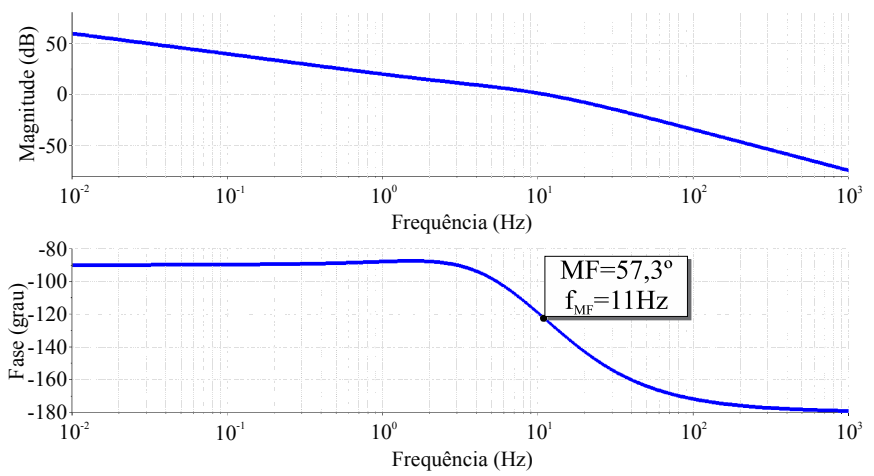

(b)

Fig. 11. (a) Resposta ao degrau unitário do sistema em malha fechada compensado (b) diagramas de Bode em malha aberta.

Cabe ressaltar que o compensador projetado e implementado é mesmo para os ambos os modos de operação, retificação e inversão.

$$
C(s)=0,2 \frac{(0,2 s+1)}{0,2 s}=0,2 \frac{(s+5)}{s} .
$$

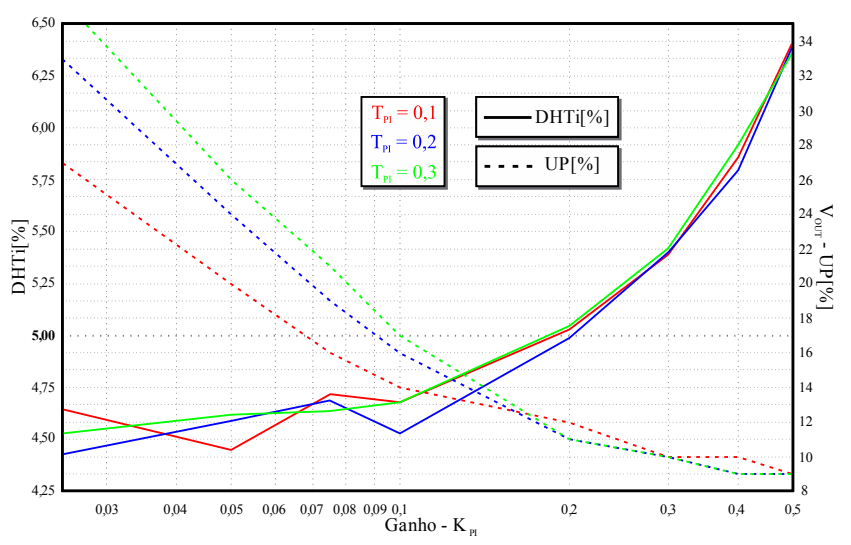

Fig. 12. Ábaco para escolha do ganho $\mathrm{K}_{\mathrm{PI}}$ e constante de tempo $\mathrm{T}_{\mathrm{PI}}$ do PI. 


\section{RESULTADOS E DISCUSSÕES}

Com a premissa de unificar o entendimento acerca da operação da estrutura de potência juntamente com o controle proposto, realizou-se algumas simulações no software PSIM ${ }^{\circledR}$ diante a variações da potência fornecida pelo conjunto de painéis fotovoltaicos $\left(\mathrm{P}_{\mathrm{PV}}\right)$, como pode ser observado na Figura 13. Por simplificação, o conversor Boost é considerado sem perdas.

De acordo com o esquema da Figura 2 e agora evidenciado também pela Figura 13.a, no modo de retificação, a corrente de entrada possui aspecto senoidal e é composta pela somatória das correntes do RNC/IPC e do SEPIC [16]. Com o aumento de $\mathrm{P}_{\mathrm{PV}}$, a corrente do RNC/IPC é atenuada para que seja possível inverter seu fluxo de potência, iniciando o modo de inversão. Neste modo de operação, cabe ao IPC assegurar um alto FP e processar todo excedente de energia.

A Figura 13.b mostra a composição dos níveis de tensão no barramento CC. Vale salientar que o controle de tensão do RHMB é realizado monitorando-se a tensão no barramento $\mathrm{CC}$ principal (composto por C1 e C2). Sendo assim, uma vez que o conversor Boost está acoplado ao mesmo capacitor $\mathrm{C} 1$ do RNC/IPC e, diferentemente do que ocorre normalmente em estruturas com inversores [22], a tensão na saída do RNC/IPC se eleva com o aumento de $\mathrm{P}_{\mathrm{PV}}$ (contribuição observada durante a retificação para satisfazer o balanço de cargas no capacitor C1). Nesse sentido, no modo de retificação, cabe ao conversor SEPIC regular a tensão no elo $\mathrm{CC}$ e, como citado anteriormente, quando sua tensão vai a zero -momento antes da inversão- o diodo D2 é polarizado diretamente, desviando assim a corrente $\mathrm{CC}$ de carga da impedância de saída do SEPIC.

A divisão do processamento de potência pode ser vista na Figura 13.c. Para a condição de intermitência da GD, isto é, $\mathrm{P}_{\mathrm{PV}}$ nula, a parcela de processamento de potência entre o RNC e o SEPIC segue a mesma proporção da composição da tensão de cada um destes em relação ao total do barramento CC, como já observado pelas Equações (2) e (3), o que representa uma potência relativa para o SEPIC de mais de $50 \%$ da potência nominal de carga. Todavia, com o aumento de $\mathrm{P}_{\mathrm{PV}}$, somente a potência do SEPIC segue uma relação direta com sua tensão de saída. Tal fato é observado pois o barramento de saída do RNC/IPC é mais sensível à injeção de potência do grupo $\mathrm{P}_{\mathrm{PV}}$ (já que a GD é alocada nesse ponto) e, uma vez que sua potência precisa se anular momentos antes de ocorrer a inversão do fluxo de potência. Cabe salientar que o dimensionamento do conversor SEPIC é realizado para a condição de intermitência, ainda que, em alguns momentos, a corrente de entrada seja composta apenas por este conversor.

Contudo, a principal característica dessa estrutura está no balanço de carga do capacitor $\mathrm{C} 1$, que tem forte relação na penetração de potência das fontes de GD. Resumidamente, as variações de potência da GD, ou até mesmo a demanda de potência de bancos de baterias, podem causar uma perturbação na tensão em C1. Diante disso, o controlador PI gera valores de $\mathrm{KV}_{\mathrm{PI}}$ a fim de estabilizar o barramento $\mathrm{CC}$, o que acaba refletindo diretamente na corrente de entrada e, por consequência, na determinação do modo de operação (retificação ou de inversão), imposto pelo comparador de histerese. Esta característica de controle, remete ao sistema descentralizado de MR CC.

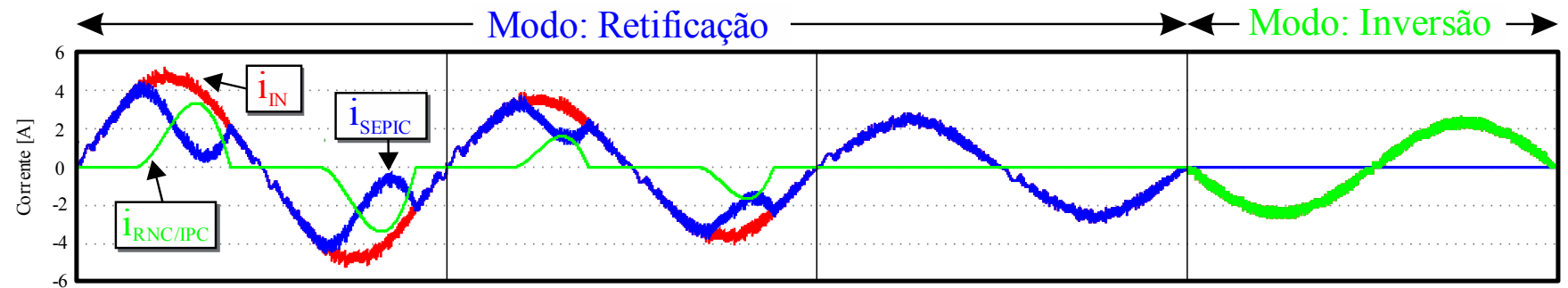

(a)

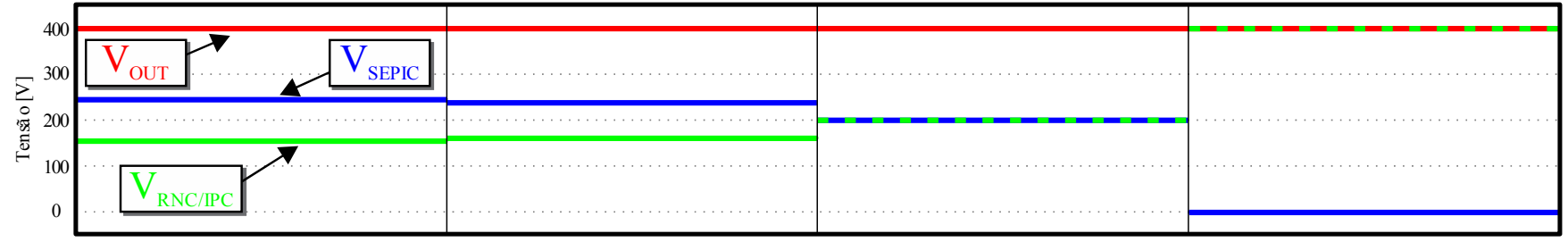

(b)

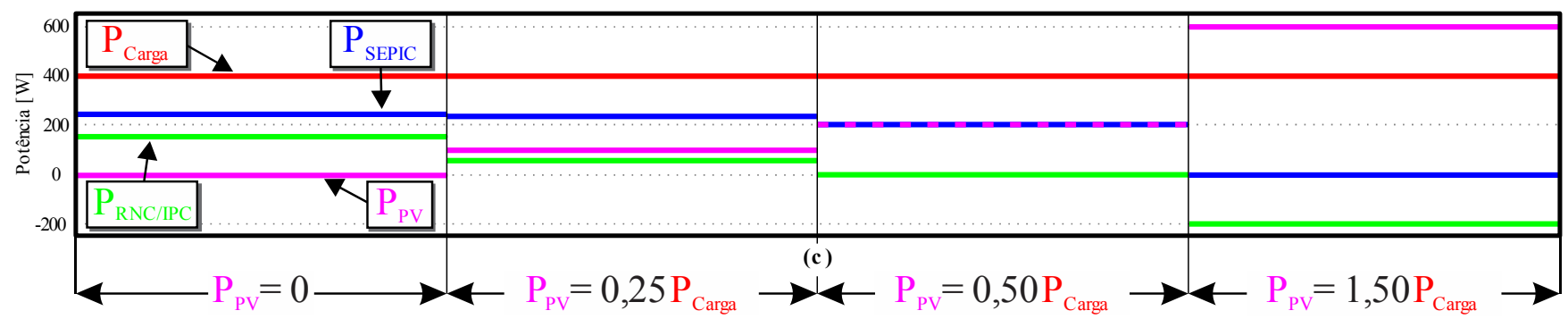

Fig. 13. Princípio operacional em diferentes regimes permanentes: (a) Composição de corrente; (b) Composição de tensão; (c) Processamento de potência. 
Com o intuito de validar o desempenho da estrutura proposta, um protótipo foi construído como ilustra a Figura 14. Os parâmetros de projeto e especificação dos componentes passivos e de semicondutores são apresentados na Tabela I. Cabe ressaltar que semicondutores com maior capacidade de condução de corrente e bloqueio de tensão foram escolhidos priorizando a robustez do protótipo implementado. Para simular o efeito de um conjunto de PV,

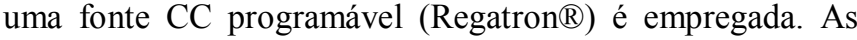
perturbações de potência desta fonte se deram por meio de degraus de irradiância capazes de fornecer uma potência maior que a de carga.

\section{TABELA I}

Especificações de Projeto

\begin{tabular}{|c|c|}
\hline Tensão de Alimentação & $127 \mathrm{Vrms}$ \\
\hline Tensão no Barramento CC & $400 \mathrm{~V}$ \\
\hline Carga & $400 \Omega / 400 \mathrm{~W}$ \\
\hline Eficiência na Retificação & Aprox. 92-93\% \\
\hline DHTi & $\begin{array}{c}\text { Em conformidade com: } \\
\text { IEC } 61000 \text { 3-2 e IEEE } 1547\end{array}$ \\
\hline \multicolumn{2}{|c|}{ Retificador Não-Controlado / Inversor Full Bridge } \\
\hline Frequência Média de Chaveamento & $25 \mathrm{kHz}$ \\
\hline L1 & $15 \mathrm{mH}$ \\
\hline $\mathrm{C} 1$ & $500 \mu \mathrm{F}$ \\
\hline Q1-Q4 & SiC SCT20N120 (20A/1200V) \\
\hline \multicolumn{2}{|c|}{ SEPIC Isolado } \\
\hline Frequência Média de Chaveamento & $25 \mathrm{kHz}$ \\
\hline Ponte Retificadora Monofásica & $35 \mathrm{~A}$ \\
\hline $\mathrm{C}_{\mathrm{EMI}}$ & $235 \mathrm{nF}$ \\
\hline $\mathrm{L} 2$ & $5 \mathrm{mH}$ \\
\hline $\mathrm{C} 2$ & $330 \mu \mathrm{F}$ \\
\hline $\mathrm{C} 3$ & $4,7 \mu \mathrm{F}$ \\
\hline D1-D2 & RHRG30120 (30A/1200V) \\
\hline Q5 & SiC SCT20N120 (20A/1200V) \\
\hline Transf. em Alta Frequência & $\mathrm{Np} / \mathrm{Ns}=39 / 50, \mathrm{Lm}=1 \mathrm{mH}$ \\
\hline \multicolumn{2}{|l|}{ Boost } \\
\hline Frequência do PWM & $25 \mathrm{kHz}$ \\
\hline L3 & $5 \mathrm{mH}$ \\
\hline $\mathrm{C}_{\mathrm{PV}}$ & $470 \mu \mathrm{F}$ \\
\hline D3 & RHRG30120 (30A/1200V) \\
\hline Q3 & SiC SCT20N120 (20A/1200V) \\
\hline
\end{tabular}

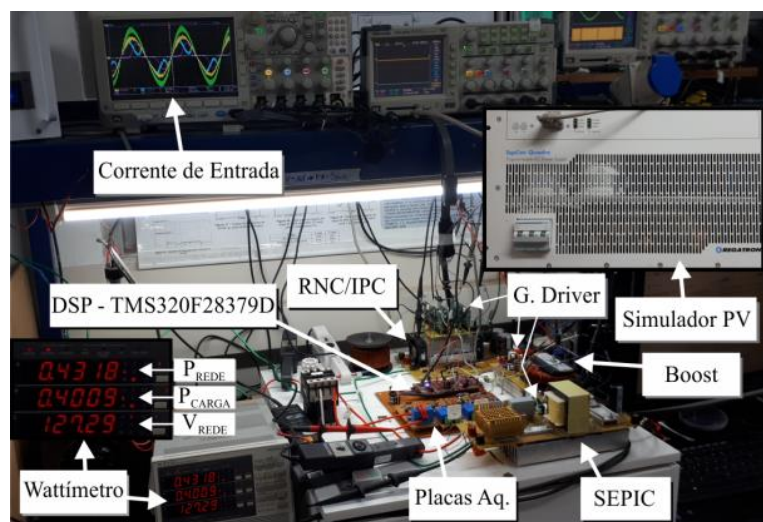

Fig. 14. Montagem experimental do RHMB.

\section{A. Composição de Corrente}

A Figura 15 mostra a composição de corrente durante a retificação com a intermitência do PV. No cruzamento da corrente de entrada por zero, o atracamento corrente-tensão é alcançado com êxito promovendo um FP próximo a unidade. Outra característica do conversor SEPIC na imposição de corrente neste período é a breve ressonância causada pelos elementos da malha L2, C3 e Lm quando o interruptor de potência do SEPIC é desativado, influenciando assim, na DHTi em baixas condições de potência. Isso ocorre, pois a frequência de chaveamento é mínima e o valor do capacitor $\mathrm{C} 3$ causa um atraso no decaimento da corrente do SEPIC. O ajuste de valores menores de capacitância é capaz de mitigar este problema.

Para as condições de projeto citadas na Tabela I, a corrente do SEPIC sempre opera no modo contínuo de condução para os níveis de tensão de entrada em 127Vrms e saída em 400V. Dessa maneira, a parcela de processamento de potência do SEPIC em relação ao RNC é majoritária. Todavia, em estruturas híbridas, quanto maior é o processamento de potência de conversores chaveados, menor é a DHTi vista pela entrada [13]. Com isso, para que o RHMB opere a níveis de tensão de alimentação de $220 \mathrm{Vrms}$ é necessário a alteração na relação de espiras do transformador do SEPIC para que seja possível a imposição de correntes com baixa DHTi.

Já para a inversão, a Figura 16 mostra a corrente de entrada defasada em $180^{\circ}$, indicando assim fluxo reverso de potência. Como a referência gerada pelo PLL é a mesma para ambos os modos (retificação e inversão), o compensador PI gera valores negativos para o controlador de corrente por histerese para o RNC/IPC. Dessa forma, o alto FP ainda é garantido. Por questões de segurança, o RHMB foi alimentado através de um varivolt e, devido à sua alta indutância de dispersão, oscilações adicionais de baixa magnitude e alta frequência são observadas tanto na tensão de alimentação, quanto na corrente drenada e injetada na rede CA (Figura 15). No modo de operação como inversor essas oscilações se mostraram mais significativas, sendo necessário reduzir a amostragem de pontos do osciloscópio (1000 pontos) a fim de eliminar os ruídos excessivos captados pelas ponteiras de tensão e corrente (Figura 16).

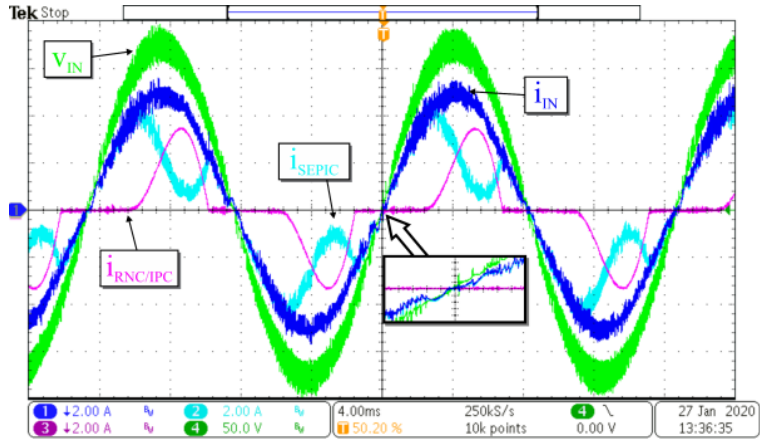

Fig. 15. Composição de corrente durante a retificação.

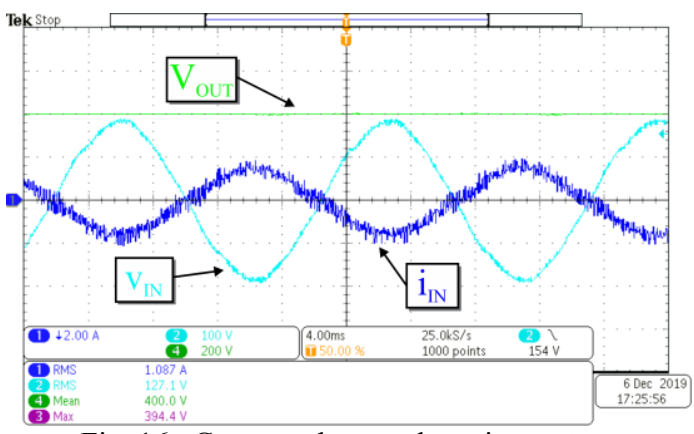

Fig. 16. Corrente de entrada na inversão. 


\section{B. Ensaios de Transição de Modo de Operação}

Como mencionado anteriormente, a validação de estabilidade da estrutura na transição entre os modos retificação-inversão se dá por meio de degraus de irradiância no simulador PV. Nesse sentido, o compensador PI é eficaz em estabilizar a tensão no barramento $\mathrm{CC}$ de saída na ocorrência de um degrau de $550 \mathrm{~W}$ de injeção de potência em $\mathrm{C} 1$ no instante "b", como mostra a Figura 17. A maior UP detectada (5\%) foi no momento de mudança do sentido da corrente de entrada. A partir do momento que ocorre de fato a transição, a tensão na saída do SEPIC é fixada com o valor negativo da tensão de polarização direta do diodo de desvio D2. É possível perceber também que o perfil de corrente na transição não apresenta picos elevados, fato este justificável pela saída do PI $\left(\mathrm{KV}_{\mathrm{PI}}\right)$ ter uma largura de banda reduzida, sendo esta a referência para a corrente de pico da entrada.

Em contrapartida, na retirada do degrau de irradiância que forneceu os 550W provenientes do PV, a estrutura retorna ao modo de retificação como ilustrado na Figura 18.

A dinâmica de imposição de corrente do SEPIC é mais rápida neste processo, enquanto que o afundamento de tensão no barramento CC de saída foi de apenas $22,09 \mathrm{~V}$, correspondendo a $5,5 \%$ do valor nominal $(400 \mathrm{~V})$. A ondulação da tensão de saída é ligeiramente maior na retificação, já que nesta condição o RNC opera em baixa frequência.

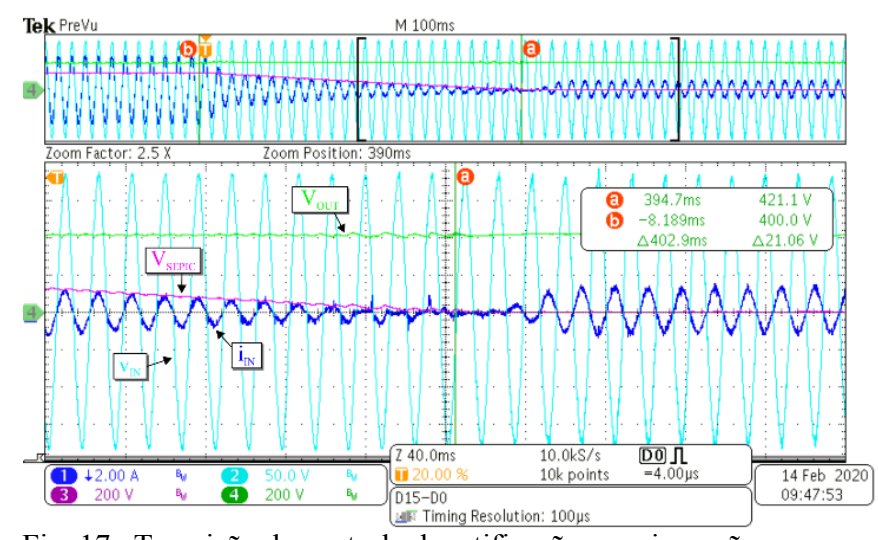

Fig. 17. Transição de controle de retificação para inversão.

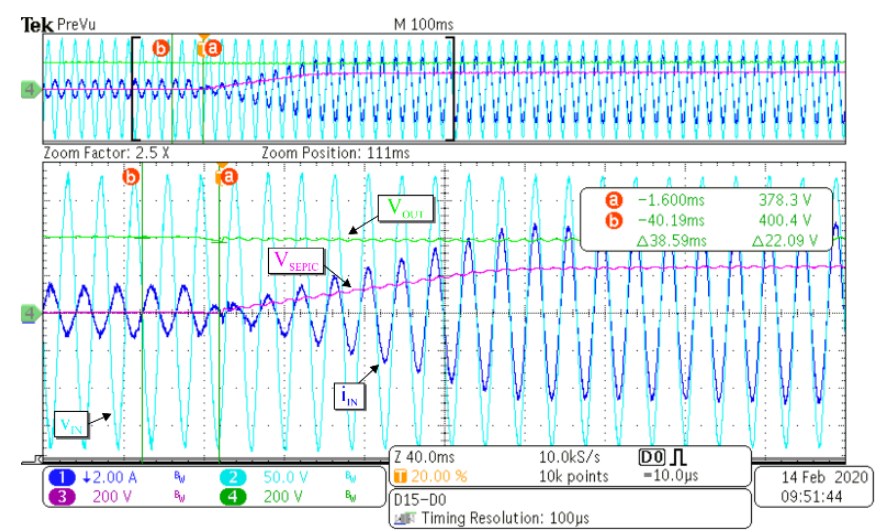

Fig. 18. Transição de controle de inversão para retificação.

\section{Resultados Qualitativos em Regime Permanente}

As curvas de rendimento, DHTi e FP apresentadas na Figura 19.a ilustram o comportamento do RHMB para ampla faixa de variação de potência entregue à carga. Adicionalmente, dois wattímetros digitais foram utilizados para monitorar a operação independente de cada conversor. $\mathrm{Na}$ Figura 19.b são apresentados os resultados obtidos em condições nominais e demonstram que o rendimento do RNC é de $96,8 \%$ e do SEPIC isolado é de $91,2 \%$. Por se tratar de um conversor SEPIC isolado em alta frequência, a parcela mais considerável de perdas pode ser atribuída ao transformador, quando comparado aos valores típicos obtidos com um conversor SEPIC não-isolado (cerca de 93\%-95\%). Observa-se ainda que, em condições nominais, cerca 35,5\% da potência entregue à carga é processada pelo RNC e 64,5\% é processada pelo conversor SEPIC isolado. Devido à contribuição do RNC, o rendimento global da estrutura cerca de 93\%- é assegurado para uma ampla faixa de variação de carga. Cabe destacar ainda, que para estruturas monofásicas, a parcela de contribuição de potência do conversor SEPIC aumenta significativamente quando comparada a estruturas trifásicas já reportadas na literatura [11], [14].

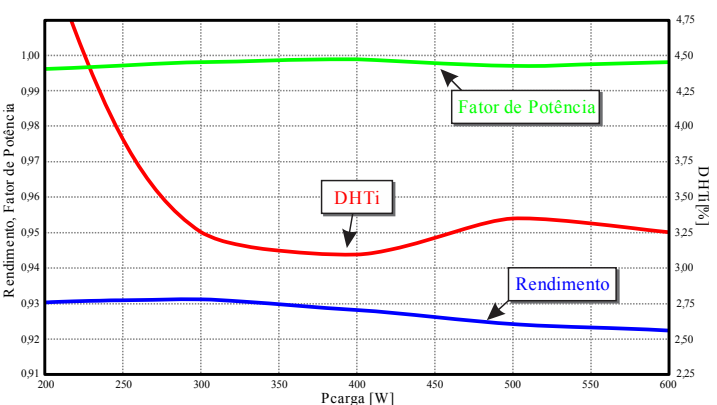

(a)
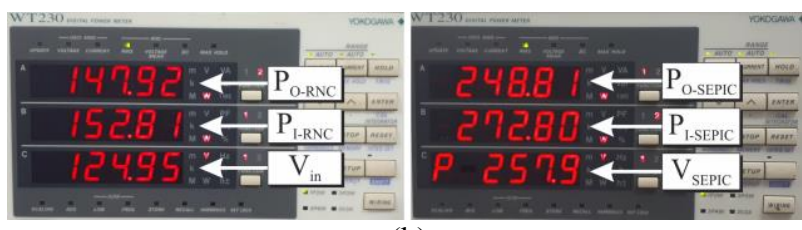

(b)

Fig. 19. Ensaios em regime permanente com o RHMB operando como retificador: (a) curvas de rendimento, DHTi e FP (b) resultados de potência processada e tensão de saída.

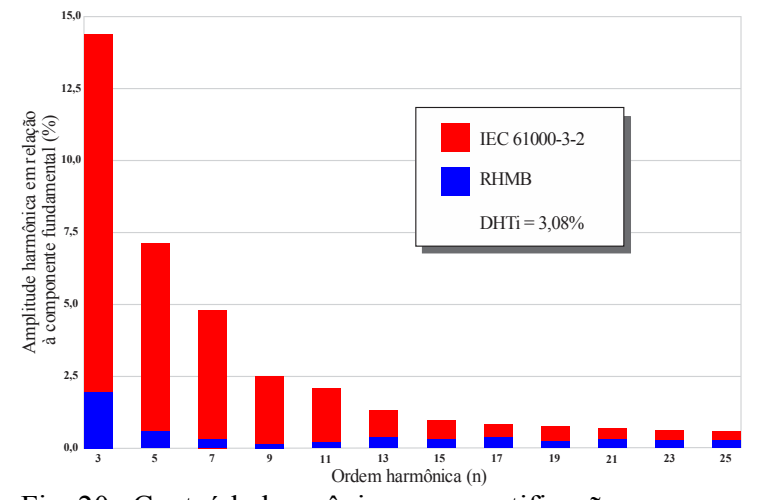

Fig. 20. Conteúdo harmônico para a retificação. 
Por fim, na Figura 20 é apresentado parte do espectro harmônico da corrente de entrada na retificação. A maior parcela de distorção está localizada na terceira harmônica devido ao comportamento já descrito da corrente em tensão nula da rede.

\section{CONCLUSÃO}

Neste trabalho foi avaliado o protótipo de uma estrutura de um retificador híbrido monofásico bidirecional (RHMB) conectado à rede capaz de compor uma microrrede CC quando houver a inserção de outras fontes alternativas de energia, tais como célula a combustível, turbina eólica etc. A utilização de um conversor Boost juntamente com um arranjo PV foi capaz de avaliar os dois modos distintos de operação da estrutura proposta (retificação e inversão), condição mandatória para aplicação em microrredes CC.

Destaca-se ainda que a estrutura proposta emprega poucos sensores e a estratégia de controle utiliza apenas um controlador PI. Isto apresenta uma grande vantagem pois garante robustez e estabilidade na transição dos modos de operação, além de facilidade no projeto.

Com relação aos parâmetros normativos, a estrutura se apresentou adequada na imposição de correntes com baixa DHTi na retificação pela IEC 61000-3-2 e no modo de inversão pela IEEE 1547. Os ensaios de transição de controle apresentaram uma dinâmica estável e com baixa UP, critério este indispensável para vida útil de equipamentos conectados à microrrede.

\section{AGRADECIMENTOS}

Os autores agradecem as agências de fomento CAPES, CNPq (sob os processos: 302945/2016-4, 303350/2019-9, 304489/2017, e 420602/2016-0) e FAPEMIG pelo suporte financeiro e ao Prof. Luiz Carlos Gomes de Freitas do NUPEP-FEELT-UFU pelas importantes contribuições na concepção e no desenvolvimento deste projeto.

\section{REFERÊNCIAS}

[1] P. H. Savage, R. R. Nordhaus, S. P. Jamieson, "DC Microgrids: Benefits and Barriers", From Silos to Systems: Issues in Clean Energy and Climate Change, vol. 26, pp. 55-60, June 2010.

[2] T. Dragičević, X. Lu, J. C. Vasquez, J. M. Guerrero, "DC Microgrids-Part II: A Review of Power Architectures, Applications, and Standardization Issues", IEEE Transactions on Power Electronics, vol. 31, no 5, pp. 3528-3549, May 2016.

[3] G. A. Xavier, "Simulação de Micro-Redes de Energia Elétrica Com Geração Fotovoltáica e Armazenamento de Energia". Dissertação de Mestrado, Universidade Federal de Viçosa, Viçosa, Brasil, 2011.

[4] M. Imecs, C. Szabo, I. I. Incze, "Modelling and Simulation of Controlled Bi-directional Power Electronic Converters in a DC Energy Distribution Line with AC Grid and Motor-Side Active Filtering”,
European Conference on Power Electronics and Applications, pp. 1-10, Sep. 2007.

[5] R. H. Lasseter, P. Piagi, "Microgrid: A Conceptual Solution", IEEE 35th Annual Power Electronics Specialists Conference, vol. 6, pp. 4285-4290, Jun. 2004.

[6] P. M. P. Batista, "Gestão Integrada de Microgeração e Armazenamento Distribuído em Redes de Distribuição BT", Dissertação de Mestrado, Faculdade de Engenharia da Universidade do Porto, Porto, Portugal, 2010.

[7] E. Rikos, S. Tselepis, C. Hoyer-Klick, M. SchroedterHomscheidt, "Stability and Power Quality Issues in Microgrids Under Weather Disturbances", IEEE Journal of Selected Topics in Applied Earth Observations and Remote Sensing, vol. 1, n 3, pp. 170179, Sep. 2008.

[8] F. Chishti, S. Mushid, B. Singh, "Development of Wind and Solar Based AC Microgrid With Power Quality Improvement for Local Nonlinear Load Using MLMS", IEEE Transactions on Industry Applications, vol. 55, $\mathrm{n}^{\circ}$ 6, pp. 7134-7145, Nov./Dez. 2019.

[9] T. Park, J. Park, H. Byun, B. Kim, C. Won, “A Droop Control with Deadbeat Current Strategy to Improve Response Characteristic for Three Phase Parallel ACDC Converter in DC Microgrid", IEEE Transportation Electrification Conference and Expo, pp. 1-6, May 2019.

[10] L. C. G. de Freitas, M. G. Simoes, C. A. Canesin, L. C. de Freitas, "Performance Evaluation of a Novel Hybrid Multipulse Rectifier for Utility Interface of Power Electronic Converters", IEEE Transactions on Industrial Electronics, vol. 54, $\mathrm{n}^{\mathrm{o}}$ 6, pp. 3030-3041, Dez. 2007.

[11] J. O. Soares, C. A. Canesin, L. C. de Freitas, F. A. S. Gonçalves, "Retificador Trifásico Híbrido Operando com Controle Digital e Modulação por Histerese", Eletrônica de Potência-SOBRAEP, vol. 13, n ${ }^{\circ} 4$, pp. 241-249, Nov. 2008.

[12] R. L. Alves, I. Barbi, "Analysis and Implementation of a Hybrid High-Power-Factor Three-Phase Unidirectional Rectifier", IEEE Transactions on Power Electronics, vol. 24, n 3, pp. 632-640, Mar. 2009.

[13] A. R. Izadinia, H. R. Karshenas, "Current Shaping in a Hybrid 12-Pulse Rectifier Using a Vienna Rectifier", IEEE Transactions on Power Electronics, vol. 33, $\mathrm{n}^{\mathrm{o}} 2$, pp. 1135-1142, Fev. 2018.

[14] A. V. Costa, D. B. Rodrigues, G. B. Lima, L. C. de Freitas, E. A. A. Coelho, V. J. Farias, L. C. G. de Freitas, "Retificador Híbrido Trifásico de Alta Potência com Reduzida DHTi Utilizando o Conversor Boost para Promover Suportabilidade a Afundamentos de Tensão", Eletrônica de Potência-SOBRAEP, vol. 17, n $\mathrm{n}^{\mathrm{O}}$ 3, pp. 609-622, Jun./Ago. 2012.

[15] C. H. I. Font, I. Barbi, "Modelagem e Controle de um Retificador Trifásico Híbrido com Elevado Fator de Potência e Capacidade de Regeneração de Energia", Eletrônica de Potência-SOBRAEP, vol. 19, no. 1, pp. 47-57, Dez. 2013/ Fev. 2014. 
[16] D. B. Rodrigues, P. R. Silva, G. B. Lima, E. A. A. Coelho, L. C. G. de Freitas, "Projeto e Análise Experimental de uma Nova Estrutura de Retificador Híbrido Trifásico Utilizando Técnica de Compensação Série de Tensão no Barramento CC", Eletrônica de Potência-SOBRAEP, vol. 23, no 3, pp. 267-280, Jul./Set. 2018.

[17] A. O. C. Neto, A. L. Soares, G. B. de Lima, D. B. Rodrigues, E. A. A. Coelho, L. C. G. de Freitas, "Optimized 12-Pulse Rectifier With Generalized Delta Connection Autotransformer and Isolated SEPIC Converters for Sinusoidal Input Line Current Imposition", IEEE Transactions on Power Electronics, vol. 34, no 4, pp. 3204-3213, Apr. 2019.

[18] T. Dragičević, X. Lu, J. C. Vasquez, J. M. Guerrero, "DC Microgrids-Part I: A Review of Control Strategies and Stabilization Techniques", IEEE Transactions on Power Electronics, vol. 31, $\mathrm{n}^{\mathrm{o}}$ 7, pp. 4876-4891, Jul. 2016.

[19] J. Schönberger, R. Duke, S. D. Round, "DC-Bus Signaling: A Distributed Control Strategy for a Hybrid Renewable Nanogrid", IEEE Transactions on Industrial Electronics, vol. 53, no 5, pp. 1453-1460, Oct. 2006.

[20] V. F. Barbosa, A. O. C. Neto, G. B. Lima, L. C. G. de Freitas, "Analysis of a Single-Phase Hybrid Bidirectional Rectifier with Series Voltage Compensation in a Grid-Connected DC Microgrid", IEEE PES Innovative Smart Grid Technologies Conference - Latin America, pp. 1-5, Sep. 2019.

[21] D. C. Martins, A. H. de Oliveira, I. Barbi, "Retificador Trifásico Isolado com Correção do Fator de Potência Empregando o Conversor CC-CC SEPIC em Condução Contínua", Eletrônica de Potência-SOBRAEP, vol. 6, $\mathrm{n}^{\mathrm{o}} 1$, pp. 08-15, Dez. 2001.

[22] C. J. O. Junior, L. P. Pires, L. C. de Freitas, E. A. A. Coelho, G. B. Lima, L. C. G. de Freitas, "Design, Analysis and Performance of a Bidirectional Solar Inverter with a Global and Independent Maximum Power Extraction Technique", IET Power Electronics, vol. 11, no 1, p 221-228, Feb. 2018.

[23] G. B. Lima, A. P. Finazzi, L. C. de Freitas, J. B. Vieira, E. A. A. Coelho, V. J. Farias, C. A. Canesin, L. C. G. de Freitas, "Single-Phase High Power Factor Hybrid Rectifier Suitable for High-Power Applications", IET Power Electronics, vol 5, $\mathrm{n}^{\mathrm{o}}$ 7, pp. 1137-1146, Aug. 2012.

[24] G. Tibola, I. Barbi "Isolated Three-Phase High Power Factor Rectifier Based on the SEPIC Converter Operating in Discontinuous Conduction Mode", IEEE Transactions on Power Electronics, vol. 28, $\mathrm{n}^{\mathrm{o}}$ 11, pp. 4962-4969, Nov. 2013.

[25] M. E. T. Souza, E. C. Resende, F. C. Melo, G. B. Lima, L. C. G. de Freitas, "Computational Implementation and Comparative Analysis of Phase-Locked Loop (PLL) Methods Under Different Power Quality Disturbances", 2019 IEEE PES Innovative Smart Grid Technologies Conference - Latin America, pp. 1-6, Sep. 2019.

[26] B. M. A. Ribeiro, L. C. G. Freitas, G. B. Lima, D. B. Rodrigues, "A Bidirectional Single-Phase Hybrid Rectifier Proposal for Sinusoidal Input Current Imposition, DC Bus Voltage Regulation and Active Power Injection onto the AC Grid", 17th International Conference on Harmonics and Quality of Power, pp. 545-550, Oct. 2016.

\section{DADOS BIOGRÁFICOS}

Vítor Fonseca Barbosa, nascido em Patrocínio (MG), em 1992, é engenheiro eletricista (2018) pela Universidade Federal do Triângulo Mineiro (UFTM) e atualmente cursa mestrado no Programa de Pós-graduação em Engenharia Elétrica (COPEL) pela Universidade Federal de Uberlândia (UFU). Suas áreas de interesse incluem: retificadores híbridos, correção ativa do fator de potência e aplicações da eletrônica de potência em microrredes CC.

Antônio de Oliveira Costa Neto, nascido em Uberlândia (MG) em 1992. Ele recebeu o grau de engenheiro eletricista e mestre respectivamente em 2015 e 2018 pela Universidade Federal de Uberlândia. Atualmente ele é doutorando do Núcleo de Pesquisa em Eletrônica de Potência. Seus interesses de pesquisa incluem retificadores híbridos aplicados em microrredes $\mathrm{CC}$, controle digital aplicado a fontes alternativas de energia e correção ativa do fator de potência.

Gustavo Brito de Lima, nascido em Ibiassucê (BA), em 1986, é engenheiro eletricista (2009), mestre (2011) e doutor (2015) pela Universidade Federal de Uberlândia (UFUFEELT). Atualmente é professor da Universidade Federal de Uberlândia. Suas áreas de interesse incluem retificadores híbridos e técnicas de controle analógicas e digitais aplicados a conversores $\mathrm{CA}-\mathrm{CC}$ e $\mathrm{CC}-\mathrm{CA}$ de interface entre fontes alternativas de energia e a rede $\mathrm{CA}$.

Danillo Borges Rodrigues, nascido em Uberlândia (MG), em 1986, é engenheiro eletricista (2011), mestre (2013) e doutor (2016) pela Universidade Federal de Uberlândia (UFU - FEELT). Atualmente é professor pela Universidade Federal do Triângulo Mineiro. Suas áreas de interesse incluem retificadores híbridos, técnicas de controle digitais, mitigação de conteúdos harmônicos de corrente, compensação de afundamentos de tensão e aplicações da eletrônica de potência em microrredes CC. 